๑ Open Access Full Text Article

\title{
A retrospective study of neoadjuvant chemotherapy plus radical hysterectomy versus radical hysterectomy alone in patients with stage II cervical squamous cell carcinoma presenting as a bulky mass
}

\author{
This article was published in the following Dove Press journal: \\ OncoTargets and Therapy \\ 13 September 2016 \\ Number of times this article has been viewed
}

\author{
Eriko Takatori \\ Tadahiro Shoji \\ Anna Takada \\ Takayuki Nagasawa \\ Hideo Omi \\ Masahiro Kagabu \\ Tatsuya Honda \\ Fumiharu Miura \\ Satoshi Takeuchi \\ Toru Sugiyama \\ Department of Obstetrics and \\ Gynecology, Iwate Medical University \\ School of Medicine, Iwate, Japan
}

Correspondence: Eriko Takatori

Department of Obstetrics and Gynecology, Iwate Medical University

School of Medicine, 19-1, Uchimaru,

Morioka, Iwate 020-8505, Japan

$\mathrm{Tel}+8 \mathrm{I} 1965 \mathrm{I} 5 \mathrm{III}$

Fax +81196221900

Email sppe8459@yahoo.co.jp
Objective: In order to evaluate the usefulness of neoadjuvant chemotherapy (NAC) for stage II cervical squamous cell carcinoma with a bulky mass, we retrospectively compared patients receiving NAC followed by radical hysterectomy (RH; NAC group) with patients who underwent RH without NAC (Ope group).

Patients and methods: The study period was from June 2002 to March 2014. The subjects were 28 patients with a stage II bulky mass in the NAC group and 17 such patients in the Ope group. The chi-square test was used to compare operative time, volume of intraoperative blood loss, use of blood transfusion, and time from surgery to discharge between the two groups. Moreover, the log-rank test using the Kaplan-Meier method was performed to compare diseasefree survival (DFS) and overall survival (OS) between the groups.

Results: There were no statistically significant differences between the two groups in operative time, volume of intraoperative blood loss, or use of blood transfusion. However, the time from surgery to discharge was 18 days (14-25 days) in the NAC group and 25 days (21-34 days) in the Ope group; the patients in the NAC group were discharged earlier $(P=0.032)$. The hazard ratio for DFS in the NAC group as compared with that in the Ope group was $0.36(95 \% \mathrm{CI}$ $0.08-0.91)$, and the 3 -year DFS rates were $81.2 \%$ and $41.0 \%$, respectively $(P=0.028)$. Moreover, the hazard ratio for OS was 0.39 (95\% CI $0.11-1.24)$, and the 3 -year OS rates were $82.3 \%$ and $66.4 \%$, respectively $(P=0.101)$.

Conclusion: NAC with cisplatin and irinotecan was confirmed to prolong DFS as compared with RH alone. The results of this study suggest that NAC might be a useful adjunct to surgery in the treatment of stage II squamous cell carcinoma presenting as a bulky mass.

Keywords: cervical cancer, neoadjuvant chemotherapy, bulky mass, prognosis

\section{Introduction}

In Japan, treatment strategies for stage II cervical carcinoma are changing. According to a report issued by the Gynecologic Tumor Committee of the Japan Society of Obstetrics and Gynecology in 2008, the number of patients who received radiotherapy or concurrent chemoradiation therapy (CCRT) without surgery was $108(33 \%)$ of 331 patients with stage IIA and 456 (50\%) of 903 patients with stage IIB. ${ }^{1}$ However, in Japan, the People's Republic of China, Italy, and several other countries, an approach employing neoadjuvant chemotherapy (NAC) has been widely used in clinical practice. ${ }^{2-4}$ In cervical carcinoma, infiltration of the parametrium and a tumor diameter of $\geq 4 \mathrm{~cm}$ are considered to be factors contributing to poor outcomes. ${ }^{5}$ Efforts to improve 
therapeutic outcomes for patients with these factors have recently become an issue.

At our institution, NAC followed by radical hysterectomy (RH) is administered to patients with a stage I/II bulky mass who provided fully informed written consent. We previously evaluated clinical outcomes of combination therapy with 3-week cycles of cisplatin (CDDP) and irinotecan (CPT-11) in a Phase II clinical trial involving patients with cervical squamous cell carcinoma in which the dose intensity of CDDP was increased in an attempt to shorten the interval to surgical therapy, the primary treatment strategy. ${ }^{6}$

Here, we evaluated the usefulness of NAC in the treatment of stage II squamous cell carcinoma presenting as a bulky mass by retrospectively comparing patients who underwent $\mathrm{RH}$ with and without NAC.

\section{Patients and methods Subjects}

Institutional Review Board approval was not deemed necessary by the Iwate Medical University School of Medicine Ethics Committee for the following retrospective review. All principles outlined in the Declaration of Helsinki were followed. Among patients with stage II cervical squamous cell carcinoma presenting as a bulky mass who had been treated between June 2002 and March 2014, the present study included 28 receiving NAC followed by $\mathrm{RH}$ (NAC group) and 17 who underwent RH alone (Ope group). At our institution, NAC is, in principle, administered to cervical carcinoma patients with a bulky mass. The Ope group consisted of the patients who did not wish to receive NAC. Specifically, the NAC group consisted of two patients at stage IIA2 and 26 at stage IIB2, while the Ope group consisted of three patients at stage IIA2 and 14 at stage IIB2. Those who had been followed up for $<6$ months were excluded. All patients received type $\mathrm{C}$ RH at our institution.

\section{NAC treatment}

CDDP at $70 \mathrm{mg} / \mathrm{m}^{2}$ was intravenously administered on day 1 , and CPT-11 at $70 \mathrm{mg} / \mathrm{m}^{2}$ was intravenously administered on days 1 and 8 of a 21-day cycle. In principle, two cycles were administered followed by RH.

\section{Inclusion criteria for NAC}

The following set of inclusion criteria was employed for the selection of study subjects: 1) histologically verified squamous cell carcinoma of the uterine cervix; 2 ) $\geq 20$ years but $<70$ years of age; 3) Eastern Cooperative Oncology Group performance status (PS), 0-2; 4) initially treated case; 5) the presence of a magnetic resonance imaging (MRI)-measurable bulky mass in the uterine cervix; 6) hematologic and blood biochemical findings meeting the following criteria: WBC count $\geq 4,000 / \mathrm{mm}^{3}$, neutrophil count $\geq 2,000 / \mathrm{mm}^{3}$, platelet count $\geq 100,000 / \mathrm{mm}^{3}$, hemoglobin $\geq 10.0 \mathrm{~g} / \mathrm{dL}$, AST and ALT levels less than or equal to two times the upper limit of normal reference range at study site, serum total bilirubin level $\leq 1.5 \mathrm{mg} / \mathrm{dL}$, serum creatinine $\leq 1.5 \mathrm{mg} / \mathrm{dL}$, and creatinine clearance $\geq 60 \mathrm{~mL} / \mathrm{min}$; 7) life expectancy $\geq 6$ months; and 8) written informed consent personally given by the subject.

\section{Exclusion criteria for NAC}

The exclusion criteria were as follows: 1) overt infection; 2) any serious complication(s), eg, cardiac disease, poorly controlled diabetes mellitus, malignant hypertension, bleeding tendency; 3) active multiple cancers; 4) interstitial pneumonia or pulmonary fibrosis; 5) effusions; 6) any history of unstable angina or myocardial infarction within 6 months after registration or a concurrent serious arrhythmia requiring treatment; 7) any contraindication for treatment with CDDP and/or CPT-11; 8) (watery) diarrhea; 9) intestinal paralysis or ileus; 10) pregnant, nursing, or wishing to become pregnant; 11) any history of serious drug hypersensitivity or drug allergy; and 12) a judgment that had been made by the attending physician that the patient was not a suitable subject for this study.

\section{Dose modification criteria}

\section{Criteria for CPT-II dose skip}

The CPT-11 dose on day 8 was to be skipped if hematologic test values within 2 days before day 8 failed to meet the following criteria: 1) neutrophil count $\geq 1,000 / \mathrm{mm}^{3}$ and 2) platelet count $\geq 75,000 / \mathrm{mm}^{3}$.

\section{Criteria for initiation of the second cycle}

Initiation of the second cycle was to be postponed up to a maximum of 2 weeks if hematologic test values within 2 days before the scheduled second cycle initiation day failed to meet the following criteria: 1) neutrophil count $\geq 1,500 / \mathrm{mm}^{3}$; 2) platelet count $\geq 75,000 / \mathrm{mm}^{3}$; and 3) serum creatinine $\leq 1.5 \mathrm{mg} / \mathrm{dL}$.

\section{Dose reduction criteria}

The doses of CDDP and CPT-11 were to be reduced to $70 \mathrm{mg} / \mathrm{m}^{2}$ and $60 \mathrm{mg} / \mathrm{m}^{2}$, respectively, during the second course in patients in whom any of the following signs of toxicity were noted in the first cycle: 1) Grade 4 neutropenia persisting for $\geq 7$ days; 2) febrile neutropenia persisting for $\geq 4$ days; 3 ) Grade 4 thrombocytopenia; 4) Grade 3 thrombocytopenia with hemorrhage; and 
5) Grade $\geq 3$ nonhematologic toxicity, excluding nausea, vomiting, appetite loss, fatigue, and hair loss.

\section{Supportive therapy}

Therapeutic administration of granulocyte-colony stimulating factor preparations was undertaken whenever Grade 4 neutropenia was noted in the first cycle. In the second cycle and thereafter, prophylactic use of this preparation in patients with Grade 3 neutropenia was acceptable, if Grade 4 neutropenia had been noted in the first cycle. Antiemetics were used prophylactically.

\section{Preoperative evaluation}

Antitumor response for the NAC group was evaluated using Response Evaluation Criteria in Solid Tumors Version 1.1 guidelines. The MRI findings at baseline were compared with the findings at the conclusion of treatment. For our efficacy evaluation, we adopted the best rating, without incorporating the response period. We also evaluated lymph node metastases by enhanced computed tomography. Adverse events were evaluated according to NCI-CTCAE Version 3.0.

\section{Postoperative adjuvant therapy}

Patients with a positive surgical margin, metastatic lymph nodes, infiltration to the parametrium, and/or vascular invasion, as demonstrated by pathological examination of the resected specimens, received postoperative irradiation, chemotherapy, or CCRT. Before 2008, CPT-11 was performed as postoperative adjuvant therapy, while chemotherapy was administered from 2008 onward. However, CCRT was performed in patients who had multiple lymph node metastases.

\section{Statistical analysis}

The two-sample $t$-test, chi-square test, and Mann-Whitney $U$-test were used to compare and analyze patient characteristics and postoperative courses in the NAC and Ope groups. Moreover, the log-rank test using the Kaplan-Meier method was performed to compare disease-free survival (DFS) and overall survival (OS) between the two groups. A $P$-value of $<0.05$ was considered to indicate a statistically significant difference.

\section{Results}

\section{Patient characteristics}

Table 1 shows the patient characteristics of the NAC and Ope groups.

\section{NAC group}

The mean age \pm SD of patients was $45.4 \pm 11.2$ years. The PS was 0 in 24 patients and 1 in four. The clinical stage was
Table I Patient characteristics

\begin{tabular}{|c|c|c|c|}
\hline Characteristics & NAC $(n=28)$ & Ope $(n=17)$ & $P$-value \\
\hline \multicolumn{4}{|l|}{$\overline{\mathrm{Age}^{\mathrm{a}}}$} \\
\hline Mean \pm SD & $45.4 \pm 11.2$ & $45.2 \pm 12.0$ & 0.973 \\
\hline \multicolumn{4}{|l|}{$\mathrm{PS}^{\mathrm{b}}$} \\
\hline 0 & 24 & 14 & 0.763 \\
\hline I & 4 & 3 & \\
\hline \multicolumn{4}{|l|}{ Stage $e^{b}$} \\
\hline Ila & 2 & 3 & 0.277 \\
\hline Ilb & 26 & 14 & \\
\hline \multicolumn{4}{|l|}{ Lymph node metastasis $^{\mathrm{b}}$} \\
\hline Positive & 7 & 12 & 0.007 \\
\hline Negative & 21 & 5 & \\
\hline \multicolumn{4}{|c|}{ Postoperative treatment ${ }^{\mathrm{b}}$} \\
\hline None & 6 & 2 & 0.432 \\
\hline Radiation & 12 & 8 & \\
\hline Chemotherapy & 9 & 5 & \\
\hline Chemoradiotherapy & I & 2 & \\
\hline
\end{tabular}

Notes: ${ }^{\mathrm{T} T w o}$-sample $t$-test. ${ }^{\mathrm{b}} \mathrm{Chi}$-square test.

Abbreviations: NAC, neoadjuvant chemotherapy; PS, performance status.

IIA2 in two patients and IIB2 in 26 . The tumor diameter, as determined by MRI before NAC, was $<5 \mathrm{~cm}$ in 13 patients and $\geq 5 \mathrm{~cm}$ in 16. All patients received two cycles of NAC and then underwent RH. According to the pathological examination of the resected specimens, seven patients were lymph node positive and 21 were lymph node negative. After surgery, radiotherapy was administered to 12 patients, chemotherapy to nine, and CCRT to one, whereas six patients did not receive postoperative adjuvant therapy.

\section{Ope group}

The mean age \pm SD of patients was $45.2 \pm 12.0$ years. The PS was 0 in 14 patients and 1 in three. The clinical stage was IIA2 in three patients and IIB2 in 14. The tumor diameter, as determined by MRI, was $<5 \mathrm{~cm}$ in nine patients and $\geq 5 \mathrm{~cm}$ in nine others. According to the pathological examination of the resected specimens, 12 patients were lymph node positive and five were lymph node negative. After surgery, radiotherapy was administered to eight patients, chemotherapy to five, and CCRT to two, whereas two patients did not receive postoperative adjuvant therapy.

\section{Treatment status with NAC}

In the $28 \mathrm{NAC}$ group patients, the antitumor responses based on the Response Evaluation Criteria in Solid Tumors Version 1.1 were complete response in four, partial response in 20 , stable disease in three, and progressive disease in one. The response rate was $85.7 \%$. NAC resulted in downstaging of the cancer in nine of the 28 patients (32.1\%). Although eleven patients $(39.3 \%)$ were diagnosed as having lymph node 
Table 2 Toxicity of NAC $(n=28)$

\begin{tabular}{|c|c|c|c|c|c|}
\hline Toxicity & Grade I, n & Grade 2, n & Grade 3, n & Grade 4, n & $\geq$ Grade $3, n(\%)$ \\
\hline \multicolumn{6}{|l|}{ Hematological } \\
\hline Leucopenia & 4 & 14 & 5 & 2 & $7(25.0)$ \\
\hline Neutropenia & 4 & 9 & 6 & 8 & $14(50.0)$ \\
\hline Anemia & 12 & 13 & 1 & 1 & $2(7.1)$ \\
\hline Thrombocytopenia & 7 & 1 & 0 & 0 & 0 \\
\hline \multicolumn{6}{|l|}{ Nonhematological } \\
\hline Nausea & 14 & 7 & 2 & 0 & $2(7.1)$ \\
\hline Vomiting & 11 & 7 & 0 & 0 & 0 \\
\hline Diarrhea & 3 & 0 & 0 & 0 & 0 \\
\hline Renal toxicity & 2 & 0 & 0 & 0 & 0 \\
\hline Neurotoxicity & 0 & 0 & 0 & 0 & 0 \\
\hline Febrile neutropenia & 0 & 0 & 2 & 0 & $2(7.1)$ \\
\hline
\end{tabular}

Notes: Adverse events were evaluated according to NCl-CTCAE Version 3.0.

Abbreviation: NAC, neoadjuvant chemotherapy.

metastasis by computed tomography before the initiation of NAC, metastasis was confirmed in the resected lymph nodes in seven patients $(25.0 \%)$.

The hematologic toxicities caused by NAC were Grade 3 and Grade 4 neutropenia in six (21.5\%) and eight $(28.6 \%)$ patients, respectively. Grade 3 or more severe anemia was observed in two patients (7.1\%), of whom one with Grade 4 anemia received a blood transfusion. No Grade 3 or more severe thrombocytopenia occurred. As for nonhematologic toxicities of at least Grade 3, two patients (7.1\%) each developed Grade 3 nausea and Grade 3 febrile neutropenia (Table 2).

In 26 of the 28 patients $(90.0 \%)$, NAC was completed as scheduled. The remaining two patients did not receive CPT-11 on day 8 of the second cycle. In these two patients, Grade 3 nausea persisted. Although their conditions did not meet the criteria for CPT-11 dose skip, their physicians decided to skip the dose. In three patients (10.7\%), the second cycle of NAC was postponed because the neutrophil counts did not meet the criteria for initiating the second cycle. However, all of these patients were able to resume treatment within 7 days. In two patients $(10.0 \%)$ with febrile neutropenia persisting for $\geq 4$ days, the doses of both CPT-11 and CDDP in the second cycle were reduced from $70 \mathrm{mg} / \mathrm{m}^{2}$ to $60 \mathrm{mg} / \mathrm{m}^{2}$.

\section{Comparison of surgical variables versus postoperative courses}

RH was performed in all 28 NAC group patients and in 17 of the Ope group patients. The operative time was 245 minutes (210-309 minutes) in the NAC group and 260 minutes (231-303 minutes) in the Ope group, and the intraoperative blood loss was $484 \mathrm{~mL}(391-857 \mathrm{~mL})$ in the NAC group and $555 \mathrm{~mL}(365-806 \mathrm{~mL})$ in the Ope group.
There were no statistically significant differences between the two groups in the operative time, the volume of intraoperative blood loss, or the use of blood transfusion. However, the time from surgery to discharge was 18 days (14-25 days) in the NAC group and 25 days (21-34 days) in the Ope group. The NAC group patients were discharged earlier $(P=0.032$; Table 3$)$.

\section{Outcome analysis}

Figures 1 and 2 show DFS and OS, respectively, analyzed by the Kaplan-Meier method. The 3-year DFS rates were 81.2\% and $41.0 \%$, respectively, in the NAC and Ope groups, with a hazard ratio (HR) of 0.36 (95\% CI 0.08-0.91). NAC significantly prolonged DFS (log-rank test, $P=0.028$ ). Moreover, the 3 -year OS rates were $82.3 \%$ and $66.4 \%$, respectively, with an HR of 0.39 (95\% CI $0.11-1.24)$. OS tended to be longer in the NAC group, though the difference did not reach statistical significance (log-rank test, $P=0.101$ ).

Table 3 Operative details

\begin{tabular}{|c|c|c|c|}
\hline Characteristics & $\begin{array}{l}\text { NAC group } \\
(n=28)\end{array}$ & $\begin{array}{l}\text { Ope group } \\
(n=17)\end{array}$ & $P$-value \\
\hline \multicolumn{4}{|l|}{ Type of surgery } \\
\hline $\mathrm{RH}$ & 28 & 17 & NA \\
\hline Other & 0 & 0 & \\
\hline \multicolumn{4}{|l|}{ Time (minutes) ${ }^{\mathrm{b}}$} \\
\hline Median (IQR) & $245(210-309)$ & $260(23 I-303)$ & 0.582 \\
\hline \multicolumn{4}{|l|}{ Blood loss $(\mathrm{mL})^{\mathrm{b}}$} \\
\hline Median (IQR) & 484 (39I-857) & $555(365-806)$ & 0.797 \\
\hline \multicolumn{4}{|l|}{ Blood transfusion ${ }^{\mathrm{a}}$} \\
\hline Yes & 4 & 3 & 0.763 \\
\hline No & 24 & 14 & \\
\hline \multicolumn{4}{|c|}{ Time from surgery to discharge (days) } \\
\hline Median (IQR) & $18(14-25)$ & $25(2 \mathrm{I}-34)$ & 0.032 \\
\hline
\end{tabular}

Notes: ${ }^{a}$ Chi-square test. ${ }^{b} M a n n-W h i t n e y ~ U$-test.

Abbreviations: NAC, neoadjuvant chemotherapy; $\mathrm{RH}$, radical hysterectomy; $\mathrm{NA}$, not applicable; IQR, interquartile range. 


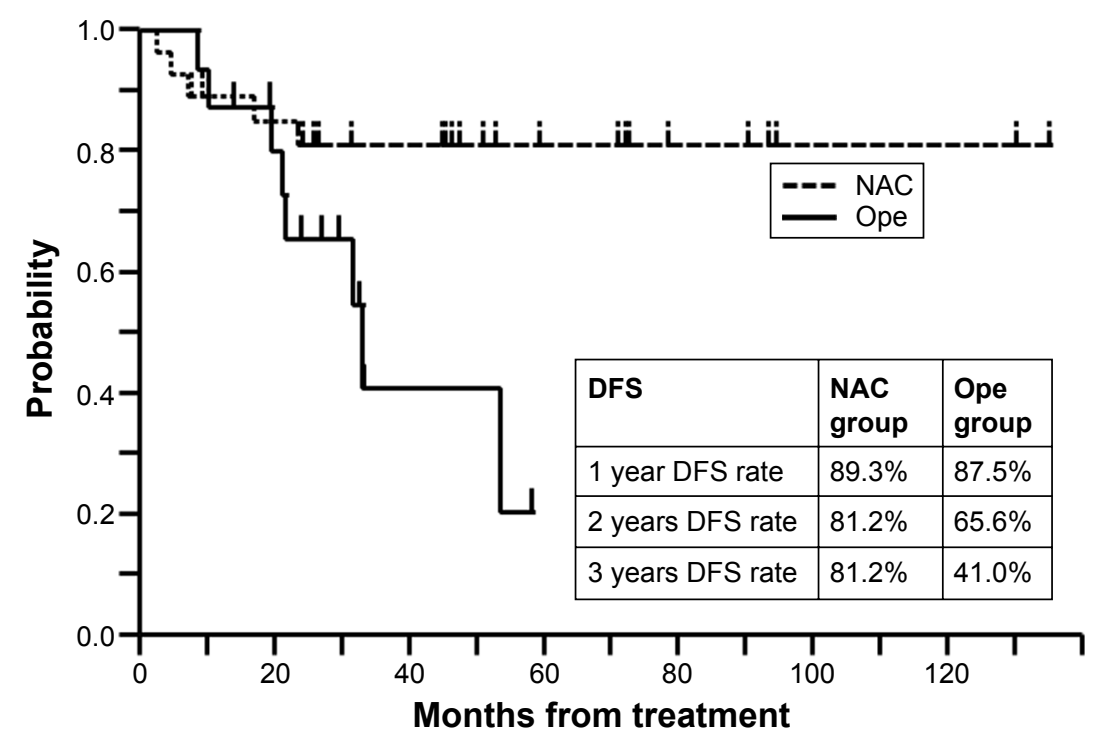

Figure I Disease-free survival.

Notes: The 3-year DFS rates were $81.2 \%$ and $41.0 \%$ in the NAC and Ope groups (without NAC), respectively. The HR was 0.36 ( $95 \%$ Cl $0.08-0.91$ ). DFS was significantly prolonged in the NAC group (log-rank test, $P=0.028$ ).

Abbreviations: DFS, disease-free survival; NAC, neoadjuvant chemotherapy; HR, hazard ratio.

\section{Discussion}

In Japan, NAC has attracted attention as a new therapy for cervical carcinoma and has been performed at many institutions since the 1990s. However, no conclusive evidence has yet been obtained about the efficacy of this strategy. In 2010, a Cochrane review analyzed six randomized controlled trials, including the Japan Clinical Oncology Group 0102 study conducted in Japan. This review reported that NAC followed by surgery did not improve OS but did improve DFS in comparison to primary surgery alone. ${ }^{7}$ Thus, NAC followed by RH can still be expected to improve the outcomes of patients. In addition, although NAC followed by RH for stage IB2 cancer has been reported to achieve good treatment outcomes, ${ }^{8}$ no conclusion has been reached on which strategy should be selected from among the options of NAC followed by $\mathrm{RH}, \mathrm{RH}$ without NAC, and CCRT for the treatment of stage IIB cancer. Furthermore, the guidelines for cervical carcinoma issued by the National Comprehensive Cancer Network and the National Cancer Institute do not contain descriptions based on histological types..$^{9,10}$ Thus, in order to

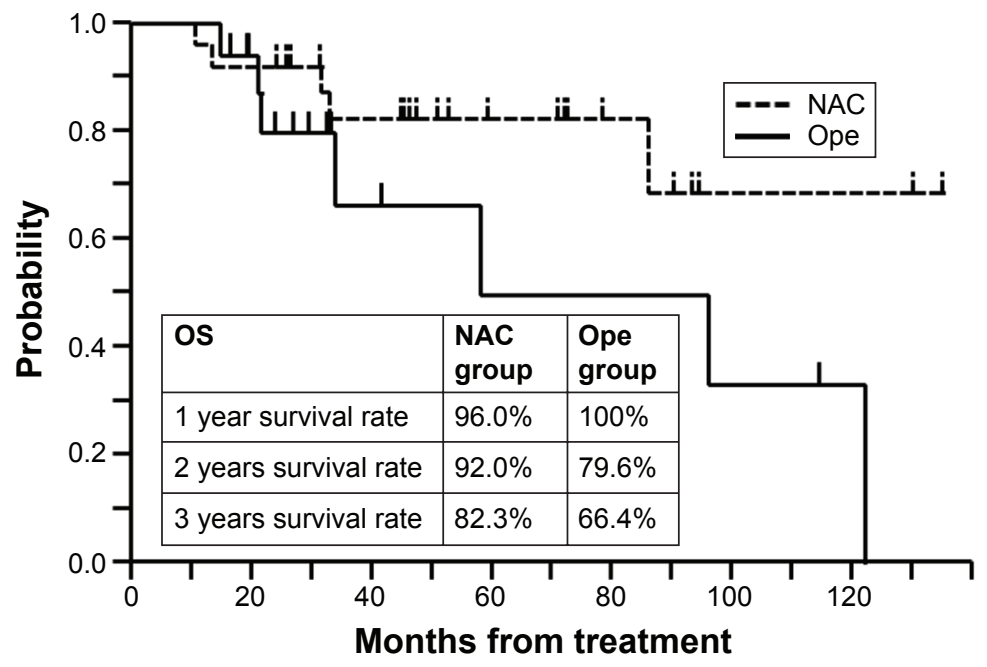

Figure 2 Overall survival.

Notes: The 3-year OS rates were $82.3 \%$ and $66.4 \%$ in the NAC and Ope groups (without NAC), respectively. The HR was 0.39 ( $95 \%$ CI 0.1 I-1.24). Although the difference did not reach statistical significance, OS tended to be prolonged in the NAC group (log-rank test, $P=0.10 \mathrm{I}$ ). Abbreviations: OS, overall survival; NAC, neoadjuvant chemotherapy; HR, hazard ratio. 
evaluate whether NAC is useful for the treatment of stage II squamous cell carcinoma presenting as a bulky mass, we compared and analyzed the treatment outcomes at our institution between the NAC and Ope groups.

At our institution, NAC is, in principle, administered to cervical carcinoma patients with a bulky mass. Thus, only patients who did not wish to receive NAC underwent $\mathrm{RH}$ alone. The NAC group had significantly prolonged DFS as compared with the Ope group $(P=0.028$; HR 0.36 [95\% CI 0.08-0.91]). Moreover, although the difference did not reach statistical significance, OS tended to be prolonged in the NAC group ( $P=0.101$; HR 0.39 [95\% CI 0.11-1.24]). Examination of the resected specimens revealed that the rate of lymph node metastasis was lower in the NAC group than in the Ope group $(P=0.001)$. This suggests that lymph node metastasis that existed before the initiation of NAC might have disappeared due to the therapy. Meanwhile, recurrence was observed in only two $(22.2 \%)$ of the nine patients in whom NAC resulted in downstaging. These factors might have contributed to prolonged DFS in the NAC group as compared to the Ope group. Because the aforementioned criteria, namely, positive surgical margin, metastatic lymph nodes, infiltration of the parametrium, and/or vascular invasion, were not met, postoperative adjuvant therapy was not administered to six patients in the NAC group and two patients in the Ope group. Among them, cancer recurred in one and two patients, respectively. In the new International Federation of Gynecology and Obstetrics classification (2009), stage IIA is further divided into substages because tumor size has been revealed to affect outcomes. ${ }^{11}$ Because large tumors are considered to be a moderate risk factor for recurrence, it was assumed that if patients who did not receive postoperative adjuvant therapy had been treated, further improvement in outcomes could have been expected. Papadia et al reported that tumor diameter is a useful information in counseling patients at the time of treatment planning with regard to the probability of being subjected to pelvic radiotherapy after completion of the initially planned treatment. ${ }^{12}$ For patients with a stage II bulky mass who received NAC, postoperative adjuvant therapy appeared to be necessary, regardless of the pathological findings of the resected specimens. While chemotherapy is administered as adjuvant therapy at an increasing number of institutions in Japan, further studies are needed to determine which regimen would be optimal if chemotherapy is administered and which modality, chemotherapy or CCRT, would achieve better treatment outcomes.
In Japan, two nationwide clinical trials have been conducted on the usefulness of NAC for the treatment of cervical carcinoma. The Japan Clinical Oncology Group 0102 study, using a BOMP regimen (consisting of bleomycin, vincristine, mitomycin-C, and CDDP) for NAC, was a randomized controlled trial that compared $\mathrm{RH}$ (plus radiotherapy) and NAC followed by RH (plus radiotherapy) in patients with a stage IB2 to IIB bulky mass. This trial was terminated when an interim analysis failed to show the usefulness of NAC. ${ }^{13}$ The Japanese Gynecologic Oncology Group 1065 study was a Phase II clinical trial conducted on NAC with nedaplatin and CPT-11 followed by $\mathrm{RH}$ in patients with a stage IB2 to IIB bulky mass. This regimen could be expected to reduce adverse events, such as nephrotoxicity and nausea/ vomiting, and was assumed to be among the useful regimens for patients with renal dysfunction as well as for the elderly in terms of quality of life. However, the response rate was $75.8 \%$, and the 2-year recurrence-free survival rate was $73.8 \%$. Because these rates were lower than the response rate to a regimen with CDDP and CPT-11, no Phase III clinical trial is currently planned. ${ }^{14}$ In addition, many negative views on NAC have been reported. ${ }^{4,15,16}$ However, these views are based on studies including patients at stage I. When the subjects are limited to patients with a bulky mass, the usefulness of NAC has been demonstrated only by a subgroup analysis limited to patients with bulky stage IB2 to IIB cancer conducted by Benedetti et al. ${ }^{3}$ Panici et al reported that type B $\mathrm{RH}$ after NAC in stage IB2 to IIB patients is a safe procedure that upholds the results of type $\mathrm{C}$, reducing operative time and late postoperative morbidity, without detrimental effect on survival. ${ }^{17}$ The usefulness of NAC has not been reported for any study including only patients with bulky stage II cancer. Treatment strategies for patients with a stage II bulky mass are an issue of considerable interest to gynecologists.

\section{Conclusion}

In patients with stage II squamous cell carcinoma presenting as a bulky mass, NAC with CDDP and CPT-11 was safely administered with no serious adverse events and achieved high treatment completion and response rates. Despite the data having been obtained in a single-center study, our analysis obtained the possibility of demonstrated prolongation of DFS, while OS was not prolonged. We can reasonably speculate that prolongation of OS might be demonstrated with further accumulation of cases. We hope that our report will lead to the initiation of a nationwide Phase II clinical trial on NAC with CDDP and CPT-11 for patients with a stage II bulky mass. 


\section{Disclosure}

The authors report no conflicts of interest in this work.

\section{References}

1. FUJINKA SHUYOUHOUKOKU. Acta Obst Gynaec JPN. 2010;62: 827-852.

2. Sugiyama T, Nishida T, Kumagai S, et al. Combination chemotherapy with irinotecan and cisplatin as neoadjuvant in locally advanced cervical cancer. Br J Cancer. 1999;81(1):95-98.

3. Benedetti-Panici P, Greggi S, Colombo A, et al. Neoadjuvant chemotherapy and radical surgery versus exclusive radiotherapy in locally advanced squamous cell cervical cancer: results from the Italian multicenter randomized study. J Clin Oncol. 2002;20(1):179-188.

4. Chen H, Liang C, Zhang L, Huang S, Wu X. Clinical efficacy of modified preoperative neoadjuvant chemotherapy in the treatment of locally advanced (stage IB2-IIB) cervical cancer: randomized study. Gynecol Oncol. 2008;110(3):308-315.

5. Bidus MA, Elkas JC; Cervical and vaginal cancer.In Berek JS, Rinehart RD editors; Berek and Novak's Gynecology. 14th edition, Lippincott Williams \& Wilkins, 2007:1403-1455.

6. Shoji T, Takatori E, Hatayama S, et al. Phase II study of tri-weekly cisplatin and irinotecan as neoadjuvant chemotherapy for locally advanced cervical cancer. Oncol Lett. 2010;1(3):515-519.

7. Rydzewska L, Tierney J, Vale CL, Symonds PR. Chemoradiotherapy for Cervical Cancer Meta-analysis Collaboration (CCCMAC). Reducing uncertainties about the effects of chemoradiotherapy for cervical cancer: individual patient data meta-analysis. Cochrane Database Syst Rev. 2010;1:CD008285.

8. Park DC, Suh MJ, Yeo SG. Neoadjuvant paclitaxel and cisplatin in uterine cervical cancer: long-term results. Int J Gynecol Cancer. 2009; 19(5):943-947.

9. Greer BE, Koh WJ, Abu-Rustum NR, et al. Cervical cancer. National Comprehensive Cancer Networks. J Natl Compr Canc Netw. 2010; 8(12):1388-1416.
10. National Cancer Institute. Cervical Cancer $\left(P D Q^{\circledR}\right)$ : Treatment, Health Professional Version. National Cancer Institute; 2016.

11. Pecorelli S. Revised FIGO staging for carcinoma of the vulva, cervix, and endometrium. Int J Gynaecol Obstet. 2009;105(2):103-104.

12. Papadia A, Bellati F, Bogani G, et al. When Does Neoadjuvant Chemotherapy Really Avoid Radiotherapy? Clinical Predictors of Adjuvant Radiotherapy in Cervical Cancer. Ann Surg Oncol. 2015; 22(Suppl 3):944-951.

13. Katsumata N, Yoshikawa H, Kobayashi H, et al. Japan Clinical Oncology Group. Phase III randomized controlled trial of neoadjuvant chemotherapy plus radical surgery vs radical surgery alone for stages IB2, IIA2, and IIB cervical cancer: a Japan Clinical Oncology Group trial (JCOG 0102). Br J Cancer. 2013;108(10):1957-1963.

14. Yamaguchi S, Nishimura R, Yaegashi N, et al. Phase II study of neoadjuvant chemotherapy with irinotecan hydrochloride and nedaplatin followed by radical hysterectomy for bulky stage Ib2 to IIb, cervical squamous cell carcinoma: Japanese Gynecologic Oncology Group study (JGOG1065). Oncol Rep. 2012;28(2):487-493.

15. Napolitano C, Imperato F, Mossa B, Framarino ML, Marziani R, Marzetti L. The role of neoadjuvant chemotherapy for squamous cell cervical cancer (Ib-IIIb): a long-term randomized trial. Eur J Gynaecol Oncol. 2003;24(1):51-59.

16. Eddy GL, Bundy BN, Creasman WT, et al. Treatment of ("bulky") stage IB cervical cancer with or without neoadjuvant vincristine and cisplatin prior to radical hysterectomy and pelvic/para-aortic lymphadenectomy: a phase III trial of the gynecologic oncology group. Gynecol Oncol. 2007;106(2):362-369.

17. Panici PB, Di Donato V, Palaia I, et al. Type B versus type C radical hysterectomy after neoadjuvant chemotherapy in locally advanced cervical carcinoma: a propensity-matched analysis. Ann Surg Oncol. 2015.
OncoTargets and Therapy

\section{Publish your work in this journal}

OncoTargets and Therapy is an international, peer-reviewed, open access journal focusing on the pathological basis of all cancers, potential targets for therapy and treatment protocols employed to improve the management of cancer patients. The journal also focuses on the impact of management programs and new therapeutic agents and protocols on

\section{Dovepress}

patient perspectives such as quality of life, adherence and satisfaction. The manuscript management system is completely online and includes a very quick and fair peer-review system, which is all easy to use. Visit http://www.dovepress.com/testimonials.php to read real quotes from published authors. 